\title{
The modelling of two DOF joints controlled by elastic inner ties
}

\author{
Dominik Ożóg ${ }^{1, *}$ \\ ${ }^{1}$ Rzeszów University of Technology, Department of Computer and Control Engineering, ul. W. Pola 2, 35-959 Rzeszów, Poland
}

\begin{abstract}
This paper considers the mathematical model of two types of joints that can be used to connect the arms of two robots. The first of them is a simple revolute joint with one degree of freedom and the second is a universal joint with two degrees of freedom. Each of them is controlled using elastic ties that run in the joints and are connected to the inside of the arm joints. This paper describes a study of kinematics, dynamics properties and Extended Denavit-Hartenberg Notation parameters mentioned joints.
\end{abstract}

\section{Introduction}

In recent years, a rapid development of the techniques related to miniaturization has been observed. This phenomenon occurs especially in the areas of electrical engineering and mechanics. The use of new ideas enables the creation of devices that are increasingly available and the improvement of current solutions, which primarily contributes to their mobility [1, 2]. However, current technological possibilities do not allow for the production of devices with the desired degree of miniaturization in many areas. Examples of such areas are medicine and structures of minimally invasive surgical robots [3]. Currently surgical robots have arms under the surface of the skin, whose diameter is 10 millimetres $[4,5]$. In the case of such redundant manipulators with many degrees of freedom, the creation of smaller structures becomes problematic. This is especially noticeable when trying to create smaller drive elements and gearboxes that drive additional joints. In addition, the power supply and control signals remain within the already installed mechanical systems in such a small space. It should be noted, that these devices must be resistant to long-term load. This is because minimally invasive surgical operations performed with such devices last much longer than classic open-patient operations. There are already electric motors for the construction of $5 \mathrm{~mm}$ diameter arms, but research at the Rzeszow University of Technology and the University of Rzeszow on gear sizes have shown a problem with the strength of the components used. The solution to these barriers can be a design that allows the installation of drive systems beyond the robot arms. By contrast, the forces generated by them are transferred to the mechanical system via flexible ties. This article presents the description of kinematics and dynamics for one joint from such a design. In this case, a universal joint with two degrees of freedom is used

\section{Universal joint with elastic inner ties}

A universal joint is a known construction commonly used as a clutch or transmission. It can also be used for manipulators, where, instead of the drive shaft, the cross connector joins two arms. In both cases, this structure has 3 degrees of freedom [6]. There are known applications of universal joints in the construction of robots. In medicine, it has been used in the construction of minimally invasive surgical robots [7]. Achieving three degrees of freedom in one joint is a great advantage that extends the work area and facilitates manoeuvring of the manipulator's arm by its user. This is very important in areas with a large number of obstacles in the work area. It is also very easy to achieve redundancy of the effector position. All it needs to do is to use 3 jointed universal joints or 2 such joints, and one with one degree of freedom.

In the present case, the tie rod is used to mutually position the manipulator arms connected by a universal joint. One of their ends is attached to the inner wall of the arm, which is further away from the base point. Through the holes in the cross connector these ties pass into the inner part of the arm closer to the base point. If the numbering of the manipulator's arms starts from the base point, the point of fitting the ties will be in the $i+1$ arm. On the other hand, after passing through the holes in the cross connector, they will be in the arm i. In this arm, the ties may be attached to the sliding mechanical constructions, which are the source of the forces acting on the arms when they are passed through the chain. Another solution is to transfer the strands outside the ith arm to apply the drive mechanisms beyond the manipulator's arms.

The second of the described solutions is the purpose of this article. The kinematics of the described solution will be approximated without specifying the drive mechanism. After further consideration, one of the ends of the tie is always fixed to the inner wall $i+1$ of the arm, while the other end of the tie is inside the i-arm. The calculation of the coordinates of the end point of the

\footnotetext{
* Corresponding author: dominikozog@,wp.p
} 
local reference system is taken into account. In this way, the anchor attachment point data can be substituted for the propulsion system regardless of whether it is within or outside of the arm.

\subsection{Kinematics for two-dimensional system}

When analyzing the universal joint in twodimensional space, it is assumed that it is possible to rotate the arms relative to each other about an axis passing through the centre of one of the cross-member bushes. Two cases of such rotation should be considered. Each case refers to another crosspiece sleeve, depending on which sleeve has rotated the axis of rotation.

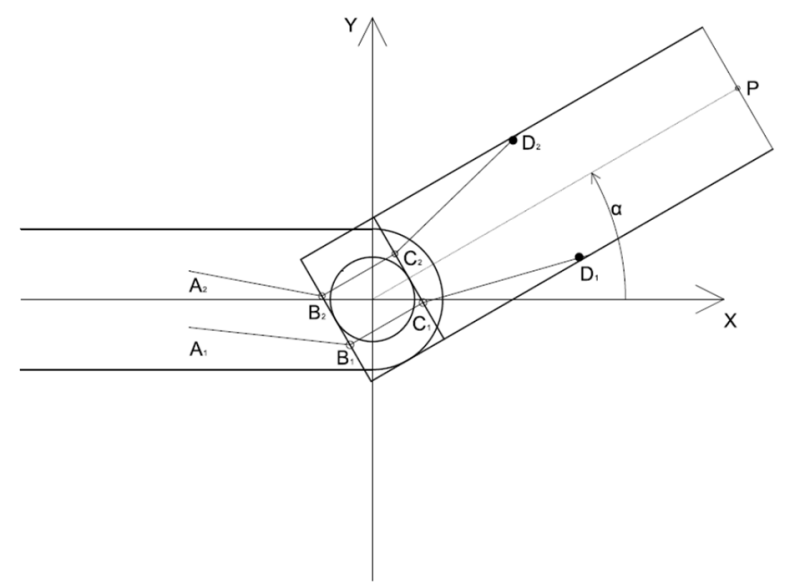

Fig. 1. Rotation around the sleeve of the cross connector connected by the i-th arm.

Fig. 1 illustrates the rotation situation around the coupling sleeve and the manipulator's arm. In this case, the sleeve rotates about the same angle as the arm $\mathrm{i}+1$.

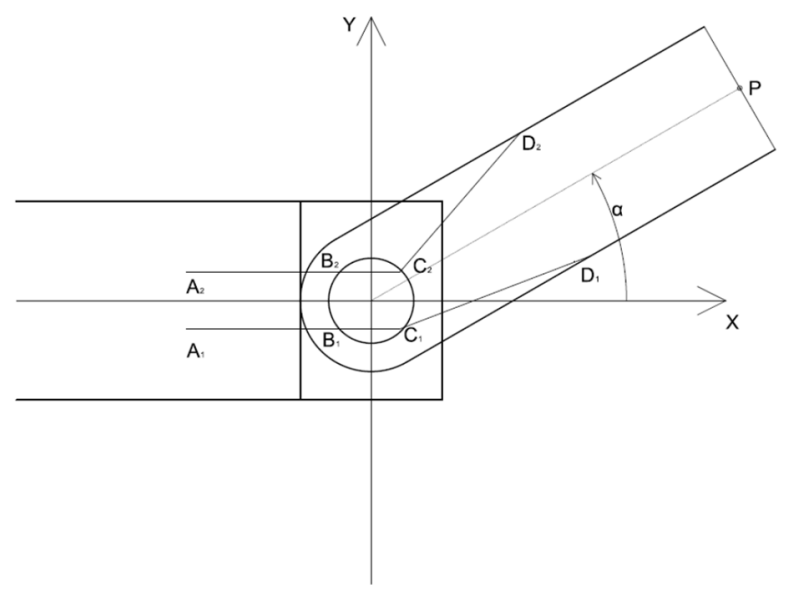

Fig. 2. Rotation around the sleeve of the cross connector connected by the $i+1 \mathrm{arm}$.

This results in a change in the length of the cable ties, between the assembly point and the drive and the cross connector sleeve. Depending on the sign of rotation angle, one of the ties will be stretched, and the other will be shortened.

In the second case (shown in Fig. 2), the axis of rotation passes through the centre of the cross-member sleeve, where the sleeve is mounted to the inner wall of the $i+1$ arm. Rotation of arm $i+1$ does not affect the sleeve. This results in a change in the length of the section between the point of attachment of the tie to the inner wall of the arm and the hole in the cross-member sleeve on the side of the arm $i+1$.

If assuming that the length of the arm $\mathrm{i}+1$ is $\mathrm{L}$ (from the centre of the joint), the position of the point $\mathrm{P}$ represents the vector:

$$
\boldsymbol{P}=\left[\begin{array}{c}
x_{P} \\
y_{P} \\
z_{P} \\
1
\end{array}\right]
$$

,where

$$
\begin{aligned}
& x_{P}=L \\
& y_{P}=0 \\
& z_{P}=0
\end{aligned}
$$

Using the Denavit-Hartenberg principle and performing actions that take into account the rotation of the Z-axis by the angle $\alpha$ we obtain the trivial task of forward and inverse kinematics [8-10]. The position of the point $\mathrm{P}$ after the transformation is given by the formula:

$$
\boldsymbol{P}^{\prime}=\left[\begin{array}{c}
L \cos (\propto) \\
L \sin (\propto) \\
0 \\
1
\end{array}\right]
$$

In turn, the equation for inverse kinematics is:

$$
\propto=\tan ^{-1}\left(\frac{y_{P \prime}}{x_{P \prime}}\right)
$$

Equations 3 and 4 are satisfied by both articulation angles (Fig. 1 and Fig. 2).

\subsection{The length of the ties as joint variables}

Equations 3 and 4 would be forward and inverse kinematics patterns for a universal joint limited to one rotation axis. However, in the case of rotation angle control by the change of the length of the ties, this length should be regarded as a pivotal variable.

The scenarios that are shown in Fig. 1 and Fig. 2 are simplified models of the system described later in this article. To simplify the equations describing them, it is assumed that the points for the position of the two ties ( $A_{i}$, $\left.B_{i}, C_{i}, D_{i}\right)$ are symmetric in relation to the axis $\mathrm{OX}$ in the initial - parallel - positioning of the arms. Point $A_{i}$ refers to the assembly point of the $\mathrm{i}$-th tie to the drive system and is defined by $A_{i}\left(x_{A i}, y_{A i}, 0\right)$ coordinates. Point $B_{i}$ is the hole in the cross connector on the side of the i-th arm. It is located at a distance $r$ from the axis OY, which is the value of the radius of the sleeve of the cross connector. This point is also at a distance $j$ from the axis OX such that $B_{i}(-$ 
$r, \pm j, 0)$. Similarly at the same distance from the OY axis, there is a point $C_{i}$ and is located in the cross-member sleeve on the side of the $\mathrm{i}+1$ arm. Point $C_{i}$ is defined as $C_{i}(r, \pm j, 0)$. Point $D_{i}$ is the place where the tie is attached to the inner wall of the $i+1$ arm. The radius of this arm is determined by $R$, and the thickness of its wall as $e$. The location of the tie is located $h$ from the OY axis in the initial position of the arms. If $t$ we define as the difference $t=R-e, D_{i}(h, \pm t, 0)$.

For a defined point, we can determine the length of the cable. Note that for the rotation around the sleeve of the cross connector connected by the i-th arm, two partial distances do not change.

$$
\begin{aligned}
& \left|B_{i}, C_{i}\right|-\text { const } . \\
& \left|C_{i}, D_{i}\right|-\text { const. }
\end{aligned}
$$

Similarly, in the rotation around the sleeve of the cross connector connected by the $i+1$ arm, however, other distances have a constant value.

$$
\begin{aligned}
& \left|A_{i}, B_{i}\right|-\text { const. } \\
& \left|B_{i}, C_{i}\right|-\text { const. }
\end{aligned}
$$

Let us define the sum of fixed values as $l_{p i}$ :

$$
\begin{aligned}
& l_{p 1}=\left|B_{i}, C_{i}\right|+\left|C_{i}, D_{i}\right| \\
& l_{p 2}=\left|A_{i}, B_{i}\right|+\left|B_{i}, C_{i}\right|
\end{aligned}
$$

For the values defined above, we know that the lengths of the ties will be denoted by the formulas:

$$
\begin{aligned}
& l_{1}=\left|A_{i}, B_{i}\right|+l_{p 1} \\
& l_{2}=\left|C_{i}, D_{i}\right|+l_{p 2}
\end{aligned}
$$

To determine the value of the inverse kinematics, determine the value of the angle $\alpha$ knowing the values of equation (8).

$$
\propto_{i}=\tan ^{-1}\left(\frac{n_{i}}{m_{i}}\right)+\tan ^{-1}\left(\frac{ \pm \sqrt{m_{i}^{2}+n_{i}^{2}-k_{i}^{2}}}{k_{i}}\right)
$$

where

$$
\begin{gathered}
k_{i}=\frac{1}{2}\left(\left(l_{i}-l_{p i}\right)^{2}-\left(z_{A i}-z_{B i}\right)^{2}-x_{A i}^{2}-y_{A i}^{2}-j^{2}-r^{2}\right) \\
m_{1}=r x_{A 1}-j y_{A 1} \\
n_{1}=j x_{A 1}+r y_{A 1} \\
m_{2}=r x_{A 2}+j y_{A 2} \\
n_{2}=-j x_{A 2}+r y_{A 2}
\end{gathered}
$$

(9) shows that $\alpha_{i}$ is a set of two angles. This results from the possibility of obtaining the same length of the tie for two different values of rotation angles with opposite characters. Since the equations for two ties are solved, the common part of the sets obtained from (9) is unambiguous:

$$
\propto=\left\{\propto \in R: \propto \in \propto_{1} \wedge \propto \in \propto_{2}\right\}
$$

Equations (2), (3), (8), (9) and (11) allow the calculation of the relationship of forward and inverse kinematics for the simplified case shown in Fig. 1. An analogous situation exists for the situation of Fig. 2 and the rotation for beta.

$$
\begin{gathered}
\beta_{i}=\tan ^{-1}\left(\frac{u_{i}}{v_{i}}\right)+\tan ^{-1}\left(\frac{ \pm \sqrt{u_{i}^{2}+v_{i}^{2}-w_{i}^{2}}}{w_{i}}\right) \\
w_{i}=\frac{1}{2}\left(\left(l_{i}-l_{p i}\right)^{2}-\left(z_{C i}-z_{D i}\right)^{2}-x_{C i}^{2}-y_{C i}^{2}-h^{2}-t^{2}\right) \\
u_{1}=-h x_{C 1}-t y_{C 1} \\
v_{1}=t x_{C 1}-h y_{C 1} \\
u_{2}=-h x_{C 2}+t y_{C 2} \\
v_{2}=-t x_{C 2}-h y_{C 2}
\end{gathered}
$$

As with the Rotation around the sleeve of the cross connector connected by the i-th arm, (12) shows the sets of two solutions. For this reason, a formula analogous to Equation (11):

$$
\beta=\left\{\beta \in R: \beta \in \beta_{1} \wedge \beta \in \beta_{2}\right\}
$$

\subsection{The length of the ties for universal joints}

In the case of a universal joint having 2 degrees of freedom, 4 ties are used. Two of them $\left(l_{1}\right.$ and $\left.l_{3}\right)$ are responsible for the rotation around the OY axis by the angle $\beta$. The next two ties $\left(l_{2}\right.$ and $\left.l_{4}\right)$ allow rotation around the axis OZ by an angle $\alpha$. The mechanism of the universal joint and the 4 ties is shown in Fig. 3

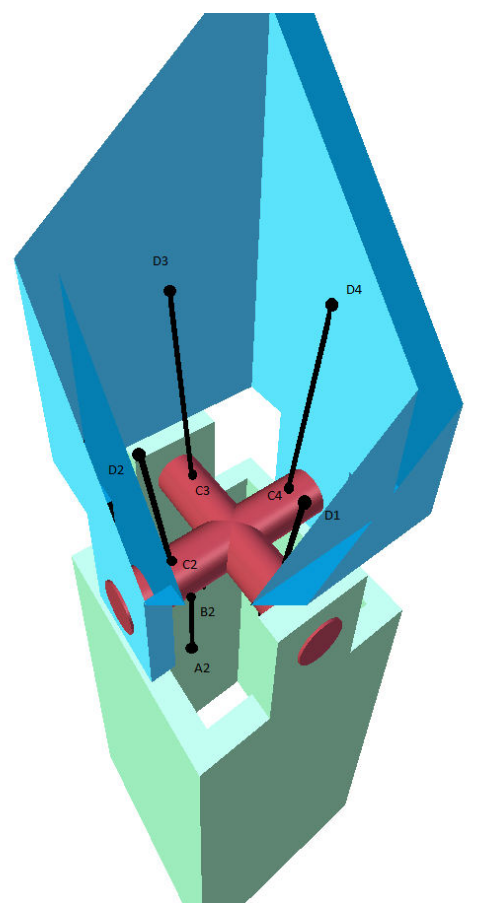

Fig. 3. Model of universal joint with 4 ties.

In Fig. 3, the points characteristic of the tie break are indicated in a manner analogous to Fig. 1 and Fig. 2. In further analysis of this paper, there is no simplification consisting in the symmetrical distribution of the puncture points. Each of these points can be placed anywhere in the joint and their coordinates are defined as $A_{i}, B_{i}, C_{i}, D_{i}$, where $i \in\{1,2,3,4\}$. 
To determine the kinematics equations for a universal joint with 2 degrees of freedom, Extended DenavitHartenberg Notation was used [11].

Table 1 lists the parameters of the universal joint controlled by the 4 ties. It included $\alpha$ and $\beta$ rotation and the length of arm $i+1$. Using the Extended DenavitHartenberg Notation formula, we obtain the transformation matrix (15).

$$
T=\left[\begin{array}{cccc}
\cos (\beta) \cos (\alpha) & -\sin (\alpha) & \sin (\beta) \cos (\alpha) & L \cos (\beta) \cos (\alpha) \\
\cos (\beta) \sin (\alpha) & \cos (\alpha) & \sin (\beta) \sin (\alpha) & L \cos (\beta) \sin (\alpha) \\
-\sin (\beta) & 0 & \cos (\beta) & -L \sin (\beta) \\
0 & 0 & 0 & 1
\end{array}\right]
$$

From the $\mathbf{T}$ matrix (15) the forward kinematics equation can be read. After their transformation and the use of trigonometric identities [12], we obtain inverse kinematics patterns for articulated variables that are values of Extended Denavit-Hartenberg Notation parameters.

$$
\begin{gathered}
\propto=\tan ^{-1}\left(\frac{y_{P}}{x_{P}}\right) \\
\beta=\tan ^{-1}\left(\frac{-z_{P}}{L \sqrt{1-\left(\frac{z_{P}}{L}\right)^{2}}}\right)
\end{gathered}
$$

Determining the length of the ties for the universal joint, the two cases shown in Fig. 1 and Fig. 2 should be taken into consideration. For the system shown in Fig. 3, where there are 4 ties, $A_{i}$ points are not subject to DenavitHartenberg conversion.

Table 1. Parameters of Extended DH-Notation.

\begin{tabular}{|c|c|c|c|c|c|}
\hline Joint & $\Theta$ & $\mathrm{d}$ & $\beta$ & $\mathrm{a}$ & $\alpha$ \\
\hline $\mathrm{i}$ & 0 & 0 & $\mathrm{var}$ & $\mathrm{L}$ & var \\
\hline
\end{tabular}

The points $B_{i}$ and $C_{i}$ are only rotated around the axis $\mathrm{OZ}$, while points $D_{i}$ are subjected to two rotations. To determine the length of ties, define $f(X, Y)$ such that:

$$
f(X, Y)=\sqrt{\sum_{i=1}\left(X_{i}-Y_{i}\right)^{2}}
$$

By using the function (17) you can define the length of the ties as:

$$
l_{i}=f\left(A_{i}, R_{Z} B_{i}\right)+f\left(R_{Z} B_{i}, R_{Z} C_{i}\right)+f\left(R_{Z} C_{i}, R_{Y} R_{Z} D_{i}\right)
$$

,where

$$
\begin{aligned}
& R_{Z}=\left[\begin{array}{cccc}
\cos (\propto) & -\sin (\propto) & 0 & 0 \\
\sin (\propto) & \cos () & 0 & 0 \\
0 & 0 & 1 & 0 \\
0 & 0 & 0 & 1
\end{array}\right] \\
& R_{Z}=\left[\begin{array}{cccc}
\cos (\propto) & -\sin (\propto) & 0 & 0 \\
\sin (\propto) & \cos () & 0 & 0 \\
0 & 0 & 1 & 0 \\
0 & 0 & 0 & 1
\end{array}\right]
\end{aligned}
$$

Arrays $A_{i}, B_{i}, C_{i}, D_{i}$ are vector-coordinated points of the corresponding points as in (1) and (3).

Constructing the inverse kinematics of the equation (18) is a very complex procedure. For this reason, it was decided to continue the work of transforming this equation by numerical methods.

\section{Dynamics of universal joints with elastic inner ties}

Determining the dynamic equations using the Lagrange method for situations of limiting the universal joint to a single rotation is a trivial task [10] and will be omitted in this document.

For further calculations, the arrangement as shown in Fig. 3 and for the axis OX directed parallel to the gravitational force, but with the opposite turn, is assumed. For these assumptions, the kinetic energy and the potential energy of the system were determined. The calculation assumed the distance from the centre of the joint to the centre of gravity of the arm i+1 equal to $L_{g}$ and the mass of that arm was equal to $M$.

Around each axis there is another force moment affecting the arm and +1 . In relation to the axis $\mathrm{OZ}$ is the force $T_{\alpha}$ and around the axis OY $T_{b}$ force. They are denominators (21) and (22).

$$
\begin{gathered}
T_{\propto}=\left(\left(\frac{d}{d t^{2}} \propto(t)\right) \cos (\beta(t))-\left(\frac{d}{d t} \propto(t)\right) \cos (\beta(t)) \sin (\beta(t))\right) M L_{g}^{2}- \\
-2 M g L_{g} \sin (\alpha(t)) \cos (\beta(t)) \\
T_{\beta}=\left(\frac{d}{d t^{2}} \beta(t)\right) M L_{g}^{2}- \\
-\left(\left(\frac{d}{d t} \beta(t)\right)^{2}-2\left(\frac{d}{d t} \alpha(t)\right)^{2}\right) M L_{g}^{2} \cos (\beta(t)) \sin (\beta(t))- \\
-2 M g L_{g} L_{g}^{2} \cos (\alpha(t)) \sin (\beta(t))
\end{gathered}
$$

\section{Conclusion}

This document shows the forward and inverse kinematics of the universal joint controlled by four elastic ties with force transmissions that can be mounted outside the robot arms. These forces affect the specific points of the manipulator's arm by the rods.

The high complexity of inverse kinematics has been demonstrated in the case of use as variable articulated lengths of ties. This problem will be the area of further research and may be solved by the use of numerical methods.

All calculations in the document concerned a single universal joint that is not associated with any further kinematic chain. The expansion of research into this area is planned in the near future.

\section{References}

1. A.B. Frazier ; R.O. Warrington ; C. Friedrich, IEEE Trans. Ind. Electron., 42, 5 (1995) 
2. M. Rasouli, L. S. J. Phee, Expert Rev Med Devices, 7, 5 (2010)

3. G. H. Ballantyne, F. Moll, Surg Clin North Am, 83, 6 (2003)

4. I. K. McLeod, P. C. Melder, Ear Nose Throat J, March 2005, 170-173 (2005)

5. K. Ikuta, T. Hasegawa, S. Daifu, ICRA '03 (2003)

6. A. J. Mazzei Jr, A. Argento, R. A. Scott, JSV 222, 1 (1999)
7. J. Shang, D. P. Noonan, C. Payne, J. Clark, M. H. Sodergren, A. Darzi, G.-Z. Yang, ICRA 2011 (2011)

8. P. I. Corke, IEEE Trans. Robot., 23, 3 (2007)

9. H. Lipkin, IDETC/CIE ASME, 7, 921-926 (2005)

10. R. Leniowski, Podstawy Robotyki, 25-84 (2005)

11. U. Thomas, F. M. Wahl, Robotic Systems for Handling and Assembly, 67, 3-16 (2010)

12. L. Rade,B. Westergren, Mathematics Handbook for Science and Engineering, 118-133 (2004) 